\title{
Urinary Bile Acid Profile of Newborns Born by Cesarean Section Is Characterized by Oxidative Metabolism of Primary Bile Acids: Limited Roles of Fetal-Specific CYP3A7 in Cholate Oxidations ${ }^{\text {}}$
}

\author{
Wen-Xia Wang, ${ }^{1}$ Li Chen, ${ }^{1}$ Guo-Yu Wang, Jin-Ling Zhang, Xian-Wen Tan, Qiu-Hong Lin, \\ Yu-Jie Chen, Jian Zhang, Ping-Ping Zhu, Jia Miao, Ming-Ming Su, Chang-Xiao Liu, Wei Jia, \\ and Ke Lan
}

Key laboratory of Drug Targeting and Drug Delivery System, Ministry of Education, West China School of Pharmacy W.-X.W., X.-W.T., Q.-H.L., Y.-J.C., J.Z., P.-P.Z., K.L.), Evidence-Based Pharmacy Center, Department of Pharmacy, West China Second University Hospital (L.C.), Labor And Delivery Room, West China Second University Hospital, (G.-Y.W., J.-L.Z.), Key Laboratory of Birth Defects and Related Diseases of Women and Children, Ministry of Education, (L.C., G.-Y.W., J.-L.Z.), and Institute of Clinical Pharmacology, West China Hospital, (J.M.), Sichuan University, Chengdu, China; Metabolomics Shared Resource, University of Hawaii Cancer Center, Honolulu, Hawaii (M.-M.S., W.J.); State Key Laboratory of Drug Delivery Technology and Pharmacokinetics, Tianjin Institute of Pharmaceutical Research, Tianjin, China (C.-X.L.); and Chengdu Health-Balance Medical Technology Co., Ltd., Chengdu, China (W.-X.W., X.W.T., Q.-H.L., Y.-J.C., J.Z., P.-P.Z., K.L.)

Received March 13, 2020; accepted April 30, 2020

\section{ABSTRACT}

This work aims to investigate how the bile acid metabolism of newborns differs from that of adults along the axis of primary, secondary, and tertiary bile acids (BAs). The total unconjugated BA profiles were quantitatively determined by enzyme digestion techniques in urine of 21 newborns born by cesarean section, 29 healthy parturient women, 30 healthy males, and 28 healthy nonpregnant females. As expected, because of a lack of developed gut microbiota, newborns exhibited poor metabolism of secondary BAs. Accordingly, the tertiary BAs contributed limitedly to the urinary excretion of BAs in newborns despite their tertiary-tosecondary ratios significantly increasing. As a result, the primary BAs of newborns underwent extensive oxidative metabolism, resulting in elevated urinary levels of some fetal-specific BAs, including 3-dehydroCA, $3 \beta, 7 \alpha, 12 \alpha$-trihydroxy-5 $\beta$-cholan-24-oic acid, $3 \alpha, 12-0 x 0-h y d r o x y-5 \beta$-cholan-24-oic acid, and nine tetrahydroxy-cholan-24-oic acids (Tetra-BAs). Parturient women had significantly elevated urinary levels of tertiary BAs and fetalspecific BAs compared with female control, indicating that they may be excreted into amniotic fluid for maternal disposition. An in vitro metabolism assay in infant liver microsomes showed that four Tetra-BAs and 3-dehydroCA were hydroxylated metabolites of cholate, glycocholate, and particularly taurocholate. However, the recombinant cytochrome $P 450$ enzyme assay found that the fetalspecific CYP3A7 did not contribute to these oxidation metabolisms as much as expected compared with CYP3A4. In conclusion, newborns show a BA metabolism pattern predominated by primary BA oxidations due to immaturity of secondary BA metabolism. Translational studies following this finding may bring new ideas and strategies for both pediatric pharmacology and diagnosis and treatment of perinatal cholestasis-associated diseases.

\section{SIGNIFICANCE STATEMENT}

The prenatal BA disposition is different from adults because of a lack of gut microbiota. However, how the BA metabolism of newborns differs from that of adults along the axis of primary, secondary, and tertiary BAs remains poorly defined. This work demonstrated that the urinary BA profiles of newborns born by cesarean section are characterized by oxidative metabolism of primary BAs, in which the fetal-specific CYP3A7 plays a limited role in the downstream oxidation metabolism of cholate.

\section{Introduction}

Bile acids (BAs) are amphipathic steroid metabolites functioning not only as agents facilitating the solubilization and absorption of lipids but also as signal molecules mediating cross talk between host and gut microbiota associated with metabolism and immunity. BAs are produced from cholesterol almost exclusively in the liver and

This work was supported partly by the Fundamental Research Funds for the Central Universities and the 111 Project of the National Ministry of Education (B18035).

${ }^{1}$ W.-X.W. and L.C. contributed equally to this work.

https://doi.org/10.1124/dmd.120.000011.

S This article has supplemental material available at dmd.aspetjournals.org. subsequently undergo extensive enterohepatic circulation driven by a series of host-gut microbial metabolism and transport mechanisms. Fundamental mechanisms for the synthesis of primary BAs in host liver and the metabolism of secondary BAs by gut microbes has been well defined in adult populations in the last century (Russell, 2003; Halilbasic et al., 2013; Dawson and Karpen, 2015). Recent works demonstrated that human CYP3A enzymes are exclusively responsible for the modification of secondary BAs into tertiary BAs, which has defined the axis of primary (host liver), secondary (gut microbiota), and tertiary (host liver) BAs in adults (Chen et al., 2019; Zhang et al., 2019). In the intestinal tract of fetus, however, there is limited colonization of bacteria responsible for secondary BA production. On the other hand, CYP3A7 rather than CYP3A4 is the major CYP3A isoform expressed in the fetal and neonatal livers (Schuetz et al., 1993; 
Kivistö et al., 1996). Therefore, the prenatal BA disposition mechanism of fetus is different from that of the adults because of a lack of secondary BA production and the downstream tertiary BA metabolism.

The difference in BA metabolism between adults and fetus has been extensively evidenced by early gas chromatography coupled with mass spectrometry (GC-MS)-based studies, in which a series of BA metabolites with "unusual" oxidation sites, including $\mathrm{C}-1 \beta,-2 \beta,-4 \beta,-6 \alpha / \beta$, and -19 , were detected and identified in early human life (Sjövall et al., 2010). Most of these studies employed chemical and/or enzymatic hydrolysis techniques to detect all BAs in their unconjugated forms because it is rather challenging to incorporate these many unconjugated, $\mathrm{N}$-amidated, sulfated, and/or glucuronidated BAs in a single bioanalytical panel. The development-related BA metabolites may be formed from cholate (CA; $3 \alpha, 7 \alpha, 12 \alpha$-trihydroxy-5 $\beta$-cholan-24-oic acid), chenodeoxycholate (CDCA; $3 \alpha, 7 \alpha$-dihydroxy-5 $\beta$-cholan-24-oic acid), deoxycholate (DCA; $3 \alpha, 12 \alpha$-dihydroxy-5 $\beta$-cholan-24-oic acid), and lithocholate (LCA; $3 \alpha$-hydroxy-5 $\beta$-cholan-24-oic acid). Some of them, particularly the hydroxylated metabolites of CA, CDCA, and DCA, were reported to have significantly higher levels in amniotic fluid, urine, and feces of pregnant women and neonatal urine, gastric contents, and feces (Almé and Sjövall, 1980; Tohma et al., 1985, 1986; Mahara et al., 1987; Setchell et al., 1988; Nakagawa and Setchell, 1990; Ikegawa et al., 1994; Kurosawa et al., 1995; Kimura et al., 1997; Meng et al., 1997b; Suzuki et al., 1997) and were also induced in patients with cholestasis, liver cirrhosis, and other liver diseases compared with healthy adults (Almé et al., 1977)(Shoda et al., 1988, 1989, 1990; Batta et al., 1989; Miyara et al., 1990; Meng et al., 1997a; Kakiyama et al., 2014). Interestingly, these oxidation sites, in addition to $\mathrm{C}-5 \beta$, have been recently characterized as CYP3A4 and CYP3A7 catalyzed tertiary oxidation sites of DCA, glycodeoxycholate, and taurodeoxycholate (Zhang et al., 2019). CYP3A7 favors the oxidations at C-19, C-4 $\beta, \mathrm{C}-6 \alpha$, $\mathrm{C}-3 \beta$, and $\mathrm{C}-1 \beta$, whereas CYP3A4 favors the oxidations at $\mathrm{C}-5 \beta$ and C-6 $\beta$ compared with each other (Chen et al., 2019). These tertiary metabolites, except for DCA-19-ol, were detected in serum and predominantly in urine of healthy adults (Zhu et al., 2018; Zhang et al., 2019).

During the past decades, the BA profiling techniques have shifted from GC-MS to liquid chromatography coupled with mass spectrometry (LC-MS) (Zhu et al., 2018). As far as we know, more than 10 years have passed since the latest GC-MS-based research focusing on the prenatal BA metabolism (Kimura et al., 1997; Maeda et al., 2003; Kumagai et al., 2007; Nishiura et al., 2010). There have been no reports on the perinatal BA metabolism using LC-MS techniques, probably because commercial sources of the authentic standards are extremely limited. Currently, it is still unclear how the fetal BA metabolism is different from that of adults along the axis of primary, secondary, and tertiary BAs. This work therefore comprehensively performed LC-MS-based BA profiling of urine samples from newborns born by cesarean section, parturient women, and healthy adults. The validated enzyme digestion-based LC-MS method was used to highlight the redox metabolism and omit the conjugation metabolism of BAs. It was confirmed that the metabolism of secondary and tertiary BAs did not contribute significantly to the BA metabolism phenotype of newborns. As a result, the urinary BA profiles of newborns are characterized by oxidative metabolism of primary BAs, which might have played a vital role in BA elimination in early life. Beyond our expectations, however, preliminary in vitro evidence showed that the fetal-specific CYP3A7 played a limited role in the downstream oxidation metabolism of CA.

\section{Materials and Methods}

Materials and Reagents. Authentic standards of 31 unconjugated BAs and four stable isotope-labeled internal standards were obtained from Steraloids (Newport, RI), TRC (Toronto, Canada), Santa Cruz (Dallas, TX), or SigmaAldrich (St. Louis, MO) as previously described (Zhu et al., 2018). Six DCA metabolites, DCA- $1 \beta$-ol, DCA- $4 \beta$-ol, DCA- $5 \beta$-ol, DCA- $6 \beta$-ol, DCA- $6 \alpha$-ol, and DCA-19-ol, were synthesized as described in our recent report (Zhang et al., 2019). Sulfatase from Helix pomatia type $\mathrm{H}-1, \beta$-glucuronidase from $H$. pomatia type $\mathrm{H}-1$, and choloylglycine hydrolase from Clostridium perfringens were purchased from Sigma-Aldrich. The urinary creatinine colorimetric assay kit was purchased from Cayman Chemical (MI). Fourteen single-donor adult liver microsomes were obtained from BD Bioscience (Woburn, MA). Eight single-donor infant liver microsomes were purchased from Corning (Corning, NY). The donor information was summarized in Supplemental Table 1. Human liver microsomes from 150 (76 female and 74 male) mixed-gender pooled donors were purchased from Corning (Tewksbury, MA). The recombinant human CYP3A4, CYP3A5, and CYP3A7 enzymes prepared from baculovirus-transfected insect cells (supersomes), NADPH Regenerating System Solution A (NADPH-A, containing $26 \mathrm{mM}$ $\mathrm{NADP}^{+}, 66 \mathrm{mM}$ glucose-6-phosphate, and $66 \mathrm{mM} \mathrm{MgCl}_{2}$ in $\mathrm{H}_{2} \mathrm{O}$ ), NADPH Regenerating System Solution B (NADPH-B, containing 40 U/ml glucose-6phosphate dehydrogenase in $5 \mathrm{mM}$ sodium citrate), and $0.5 \mathrm{M}$ PBS ( $\mathrm{pH} 7.4$ ) were purchased from Corning. Calcium chloride $\left(\mathrm{CaCl}_{2}, 96 \%\right)$ was purchased from Tianjin Kemiou Chemical (China). LC-MS-grade methanol, acetonitrile, and formic acid were purchased from Sigma-Aldrich. DMSO was purchased from Thermo Fisher Scientific (Waltham, MA). Ultrapure water was obtained by using a Milli-Q system (Millipore, Bedford).

Human Urine Samples. Overnight fasting urine samples were collected at Institute of Clinical Pharmacology, West China Hospital of Sichuan University, from 58 healthy volunteers comprising 30 males and 28 females. All urine samples were stored at $-80^{\circ} \mathrm{C}$ until analysis. Briefly, the inclusion criteria for healthy subjects were normal blood, liver, and kidney functions; negative test results for the biomarker of infectious diseases, including hepatitis $\mathrm{B}$, hepatitis $\mathrm{C}$, human immunodeficiency virus, and Treponema pallidum; no abnormalities in electrocardiogram, abdominal ultrasonography, and chest radiography; no history of gastrointestinal surgery except for appendicectomy; and no ingestion of any medications or dietary supplements 2 weeks before urine collections. Pregnancy was excluded for all females by serum human chorionic gonadotropin test. The demographic information of the healthy adult population enrolled in this work is listed in Supplemental Table 2. The studies of BAs in healthy adult urine samples were approved by the Institutional Review Board of West China Hospital of Sichuan University.

Twenty-nine healthy parturient women were recruited at the Labor and Delivery Room of West China Second University Hospital of Sichuan University. Medicinal history of routine prenatal examinations was checked to exclude those with abnormalities during pregnancy, including gestational diabetes mellitus, 
intrahepatic cholestasis of pregnancy, gestational hypertension, iron deficiency anemia during pregnancy, etc. Urine samples of the parturient women were collected from catheterization before cesarean section. All neonates were delivered by cesarean section, and their diaper disposals were collected as much as possible within 48 hours of birth. Diaper samples without contamination of meconium were collected from 21 newborns, stored in an ice box, and transferred to the bioanalytical laboratory. The urine samples of newborns that were absorbed by diaper gel were isolated with the protocol described below. All urine samples were stored at $-80^{\circ} \mathrm{C}$ until analysis. The demographic information of pregnant women and newborns enrolled in this work are listed in Supplemental Tables 2 and 3. The studies of BAs in urine samples of pregnant women and newborns were approved by the Institutional Review Board of Sichuan University.

Validation of Diaper Urine Isolation Protocol. We have tested two methods to isolate urine samples that were absorbed by diaper gel. The methanol method was to process the diaper gel with five folds (v/w) of methanol by overnight sonication. The $\mathrm{CaCl}_{2}$ method was to process the gel with $2 \%$ (w/w) $\mathrm{CaCl}_{2}$ according to Hu et al. (2004) and Liu et al. (2014). The BAs extracted from diaper gel were determined with the enzyme digestion method described below, and the recoveries were calculated in comparison with the direct analysis of a pooled urine sample of in-laboratory volunteers. As shown in Supplemental Table 4, extraction recoveries of the time-consuming methanol method for most BAs were below $50 \%$. However, the data from the $\mathrm{CaCl}_{2}$ method were within $80 \%-110 \%$ and remained stable during multiple tests except for the most hydrophobic LCA $(64 \% \pm 9 \%, n=4)$. An additional test of creatinine confirmed that the $\mathrm{CaCl}_{2}$ method may extract urinary creatinine from diaper gel with recoveries of $95 \% \pm 4 \%(n=8)$. The $\mathrm{CaCl}_{2}$ method was therefore employed in this work. Briefly, the diaper urine was released and separated by adding $\mathrm{CaCl}_{2}(2 \%$, w/w) into the wet gel, vortex mixed at $1500 \mathrm{rpm}$ for 10 minutes, and centrifuged at $10^{\circ} \mathrm{C}$ at $600 \mathrm{~g}$ for 10 minutes.

Urinary Creatinine Assay. Urinary creatinine levels were measured by the alkaline picrate method with kits purchased from Cayman Chemical. The absorbance value at $490 \mathrm{~nm}$ was measured before and after acid addition, and the difference between the absorbance was used to calculate the creatinine levels according to the creatinine standard curve.

Sample Preparation for Unconjugated BA Profiling. Analysis of BA profiles was performed using the enzyme digestion techniques published in our recent work (Zhu et al., 2018). Following the strategy employed by the GC-MS-based BA analysis, this method was designed and validated to detect all BAs in their unconjugated forms. Information of the $N$-amidated, sulfated, and/or glucuronidated BAs was accordingly lost in this work. First, $50 \mu \mathrm{l}$ of urine sample was transferred to a 700- $\mu$ l round-well 96-well plate. Second, $150 \mu \mathrm{l}$ of sodium acetate buffers ( $\mathrm{pH} 5.0$ ) containing $100 \mathrm{U}$ of cholylglycine hydrolase, $50 \mathrm{U}$ of sulfatase, and $500 \mathrm{U}$ of $\beta$-glucuronidase were added to hydrolyze the $N$-acylamidated, glucuronidated, and/or sulfated forms into the unconjugated forms. The calibration and quality control samples were prepared with $150-\mu 1$ buffers without any enzymes. The plate was incubated at $37^{\circ} \mathrm{C}$ for 6 hours and subsequently lyophilized. Two hundred microliters of acetonitrile containing $1 \%$ formic acid and $100 \mathrm{nM}$ internal standards (LCA-2,2,4,4-D4, DCA-2,2,4,4-D4, UDCA-2,2,4,4-D4, and CA-2,2,4,4-D4) was added in each well. The plate was vortex mixed at $1500 \mathrm{rpm}$ at $10^{\circ} \mathrm{C}$ for 30 minutes and centrifuged at $4^{\circ} \mathrm{C}$ at $3000 \mathrm{~g}$ for 20 minutes. Two hundred microliters of supernatant was vacuum evaporated at $30^{\circ} \mathrm{C}$. The residue was reconstituted with $50 \mu \mathrm{l}$ acetonitrile and $50 \mu \mathrm{l}$ water by vortex mixing at $900 \mathrm{rpm}$ for 20 minutes. After centrifugation, the plate was placed into autosampler for subsequent analysis.

In Vitro Metabolism Assay. In vitro metabolism was performed using the protocol described in our recent report (Chen et al., 2019; Zhang et al., 2019). In brief, stock solutions of BAs were prepared in DMSO. Incubations with initial substrate concentrations of 1.0, 5.0, 25, 50, 100, 200, and $300 \mu \mathrm{M}$ were performed for DCA in 96 -well plates at $37^{\circ} \mathrm{C}$ to acquire the oxidation kinetic data. Incubations of CA, taurocholate (TCA), and glycocholate (GCA) were comparatively performed at an initial substrate concentration of $50 \mu \mathrm{M}$. The 100- $\mu$ l incubation solution contained 0.1 M PBS ( $\mathrm{pH} 7.4), 5.0 \mu \mathrm{l}$ of NADPH-A, $1.0 \mu \mathrm{l}$ of NADPH-B, $0.5 \mu \mathrm{l}$ of BA working solution, $0.5 \mu \mathrm{l}$ of blank solvent, and $2.5 \mu \mathrm{l}$ of liver microsomes (protein concentration of $20 \mathrm{mg} / \mathrm{ml}$ ). The final protein concentration in the incubation media was $0.5 \mathrm{mg} / \mathrm{ml}$. All incubations were performed in triplicate, and the DMSO concentration in all incubations was $1 \%(\mathrm{v} / \mathrm{v})$. The reactions were initiated after 5 minutes of preincubation at $37^{\circ} \mathrm{C}$ by adding liver microsomes and stopped at 60 minutes after incubation by adding $300 \mu \mathrm{l}$ of ice-cold acetonitrile containing $0.1 \%$ formic acid and $50 \mu \mathrm{M}$ CA-2,2,4,4-D4. The reaction mixture was then centrifuged at $4^{\circ} \mathrm{C}$ at $4000 \mathrm{~g}$ for 20 minutes. For incubations using unconjugated BA as substrates, the supernatant $(50 \mu \mathrm{l})$ was diluted with an equal volume of water and subjected to LC-MS/MS analysis. For incubations using TCA or GCA as substrates, the supernatant was vacuum evaporated and subsequently prepared according to the enzyme digestion technique described above to hydrolyze the metabolites into unconjugated forms.

Quantitative LC-MS/MS Analysis. Quantitative analysis of unconjugated BAs was performed using a previously described method with a slightly modified gradient on ACQUITY ultraperformance liquid chromatography coupled to a Xevo TQ-S mass spectrometer (Waters, Milford, MA) (Zhu et al., 2018). The mobile phases consisted of $0.01 \%$ formic acid in water (mobile phase A) and acetonitrile. Five microliters of each sample was injected onto an ACQUITY BEH C18 column $(1.7 \mu \mathrm{m}, 100 \mathrm{~mm} \times 2.1 \mathrm{~mm})$ (Waters). The flow was $0.45 \mathrm{ml} / \mathrm{min}$ with a 16-minute gradient: $0.0-0.5$ minutes $(95 \% \mathrm{~A}), 0.5-1.0$ minute $(95 \%-60 \% \mathrm{~A})$, 1.0-2.0 minutes (60\%-74\% A), 2.0-4.0 minutes (74\%-70\% A), 4.0-6.0 minutes (70\% A), 6.0-7.0 minutes (70\%-62\% A), 7.0-11.0 minutes $(62 \%-60 \% \mathrm{~A})$, 11.0-13.5 minutes (60\%-20\% A), 13.5-14.0 minutes (20\%-0\% A), 14.0-15.0 minutes (0\% A), and 15.0-16.0 minutes (0\%-95\% A). The mass spectrometer was operated in the negative mode with a $3.0-\mathrm{kV}$ capillary voltage. The source and desolvation temperatures were set at $150^{\circ} \mathrm{C}$ and $550^{\circ} \mathrm{C}$, respectively. Nitrogen and argon were used as cone and collision gases, respectively. The cone gas flow and desolvation gas flow were set at 150 and $950 \mathrm{l} / \mathrm{h}$, respectively. Selected ion recording for quantification and identification of unconjugated BAs was described in our previous reports (Yin et al., 2017; Zhu et al., 2018).

Data Processing. The LC-MS/MS raw data were processed by UNIFI (V1.8; Waters). The concentration of urinary BA levels in individuals was corrected by creatinine levels and expressed as nanomoles per millimoles creatinine. Multivariate analysis of the creatinine-adjusted urinary BA profiles was carried out by using SIMCA P+ (version 13; Umetrics, Umeå, Sweden). Unpaired $t$ tests of the creatinine-adjusted urinary levels were performed between populations by using SPSS 21.0 (IBM-SPSS Inc., IL). The in vitro metabolite formations as a function of substrate concentrations were fit to Hill equation using GraphPad Prism software (version 7.0; GraphPad Software, La Jolla, CA).

\section{Results}

Newborns Have Urinary BA Profiles Markedly Different from Adults. A total of $40 \mathrm{C} 24$ BAs were detected in urine samples of the tested populations by using the enzyme digestion-based LC-MS method. The representative ion chromatograms of the digested urine samples are shown in Supplemental Fig. 1. Conjugation patterns of the detected BAs were not investigated in this work. Among the detected BAs, 31 species were quantified with the in-laboratory authentic standards, and nine unidentified tetrahydroxy-cholan-24-oic acids (Tetra-BA-1 9) were semiquantitatively determined with the calibration curve of CA. As shown in Fig. 1, the downstream metabolism of CDCA produced a total of 13 known metabolites, and the downstream metabolism of CA produced a total of 18 known metabolites. Figure $2 \mathrm{~A}$ showed the PCA scores plot of the creatinine-adjusted BA profiles. The PCA analysis found a poor separation between healthy adults and pregnant women, however, both groups demonstrated a clear separation from newborns. The OPLS-DA analysis confirmed the poor separation between healthy men and women (Fig. 2B), an acceptable separation between pregnant women and healthy adults (Fig. 2C), and strong separations between newborns and healthy adults (Fig. 2D) or between newborns and pregnant women (Fig. 2E).

The creatinine-adjusted urinary levels of the representative BAs detected in the tested populations are illustrated in detail in Fig. 3, in which they were categorized in one way into primary, secondary, and 


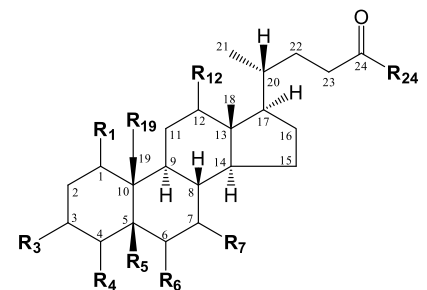

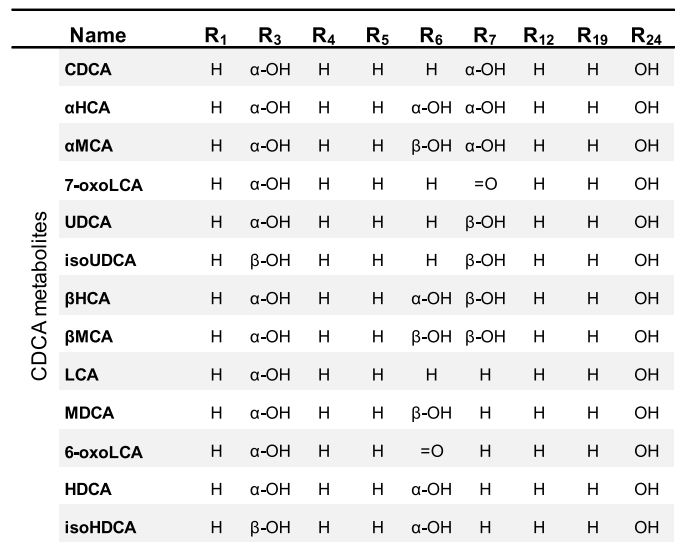

\begin{tabular}{llllllllllll}
\hline CA & $H$ & $a-O H$ & $H$ & $H$ & $H$ & $a-O H$ & $a-O H$ & $H$ & $O H$
\end{tabular}

\begin{tabular}{|c|c|c|c|c|c|c|c|c|c|}
\hline 3-dehydroCA & $\mathrm{H}$ & $=0$ & $\mathrm{H}$ & $\mathrm{H}$ & $\mathrm{H}$ & $\alpha-\mathrm{OH}$ & $a-O H$ & $\mathrm{H}$ & $\mathrm{OH}$ \\
\hline isocA & $\mathrm{H}$ & $\beta-\mathrm{OH}$ & $\mathrm{H}$ & $\mathrm{H}$ & $\mathrm{H}$ & $\alpha-\mathrm{OH}$ & $\mathrm{a}-\mathrm{OH}$ & $\mathrm{H}$ & $\mathrm{OH}$ \\
\hline 7-oxoDCA & H & $\alpha-\mathrm{OH}$ & H & $\mathrm{H}$ & $\mathrm{H}$ & $=0$ & $\alpha-\mathrm{OH}$ & $\mathrm{H}$ & $\mathrm{OH}$ \\
\hline UCA & $\mathrm{H}$ & $\alpha-\mathrm{OH}$ & $\mathrm{H}$ & $\mathrm{H}$ & $\mathrm{H}$ & $\beta-\mathrm{OH}$ & $a-O H$ & $\mathrm{H}$ & $\mathrm{OH}$ \\
\hline isoUCA & $\mathrm{H}$ & $\beta-\mathrm{OH}$ & $\mathrm{H}$ & $\mathrm{H}$ & $\mathrm{H}$ & $\beta-\mathrm{OH}$ & $a-O H$ & $\mathrm{H}$ & $\mathrm{OH}$ \\
\hline 12-oxoCDCA & $\mathrm{H}$ & $\alpha-\mathrm{OH}$ & $\mathrm{H}$ & $\mathrm{H}$ & $\mathrm{H}$ & $a-\mathrm{OH}$ & $=0$ & $\mathrm{H}$ & $\mathrm{OH}$ \\
\hline 12-epicA & $\mathrm{H}$ & $\alpha-\mathrm{OH}$ & $\mathrm{H}$ & $\mathrm{H}$ & $\mathrm{H}$ & $\alpha-\mathrm{OH}$ & $\beta-\mathrm{OH}$ & $\mathrm{H}$ & $\mathrm{OH}$ \\
\hline DCA & $\mathrm{H}$ & $\alpha-\mathrm{OH}$ & $\mathrm{H}$ & $\mathrm{H}$ & $\mathrm{H}$ & $\mathrm{H}$ & $\alpha-\mathrm{OH}$ & $\mathrm{H}$ & $\mathrm{OH}$ \\
\hline isoDCA & $\mathrm{H}$ & $\beta-\mathrm{OH}$ & $\mathrm{H}$ & $\mathrm{H}$ & H & $\mathrm{H}$ & $\alpha-\mathrm{OH}$ & $\mathrm{H}$ & $\mathrm{OH}$ \\
\hline 12-oxoLCA & $\mathrm{H}$ & $\alpha-\mathrm{OH}$ & $\mathrm{H}$ & $\mathrm{H}$ & $\mathrm{H}$ & $\mathrm{H}$ & $=0$ & $\mathrm{H}$ & $\mathrm{OH}$ \\
\hline 12-еріDCA & $\mathrm{H}$ & $\alpha-\mathrm{OH}$ & $\mathrm{H}$ & $\mathrm{H}$ & H & H & $\beta-\mathrm{OH}$ & $\mathrm{H}$ & $\mathrm{OH}$ \\
\hline DCA-19-ol & $\mathrm{H}$ & $\alpha-\mathrm{OH}$ & $\mathrm{H}$ & $\mathrm{H}$ & $\mathrm{H}$ & $\mathrm{H}$ & $\mathrm{a}-\mathrm{OH}$ & $\mathrm{OH}$ & $\mathrm{OH}$ \\
\hline DCA-6ß-ol & $\mathrm{H}$ & $\alpha-\mathrm{OH}$ & $\mathrm{H}$ & $\mathrm{H}$ & $\beta-\mathrm{OH}$ & $\mathrm{H}$ & $\mathrm{a}-\mathrm{OH}$ & $\mathrm{H}$ & $\mathrm{OH}$ \\
\hline DCA- $5 \beta-$ ol & $\mathrm{H}$ & $\alpha-\mathrm{OH}$ & $\mathrm{H}$ & $\mathrm{OH}$ & H & $\mathrm{H}$ & $\alpha-\mathrm{OH}$ & $\mathrm{H}$ & $\mathrm{OH}$ \\
\hline DCA-6a-ol & $\mathrm{H}$ & $\alpha-\mathrm{OH}$ & $\mathrm{H}$ & $\mathrm{H}$ & $\alpha-\mathrm{OH}$ & $\mathrm{H}$ & $\alpha-\mathrm{OH}$ & $\mathrm{H}$ & $\mathrm{OH}$ \\
\hline DCA- $1 \beta-0 \mid$ & $\beta-\mathrm{OH}$ & $\alpha-\mathrm{OH}$ & $\mathrm{H}$ & $\mathrm{H}$ & $\mathrm{H}$ & $\mathrm{H}$ & $\alpha-\mathrm{OH}$ & $\mathrm{H}$ & $\mathrm{OH}$ \\
\hline DCA- $4 \beta-01$ & $\mathrm{H}$ & $\alpha-\mathrm{OH}$ & $\beta-\mathrm{OH}$ & $\mathrm{H}$ & $\mathrm{H}$ & $\mathrm{H}$ & $\alpha-\mathrm{OH}$ & $\mathrm{H}$ & $\mathrm{OH}$ \\
\hline
\end{tabular}

Primary BA Secondary BA $\square$ Tertiary BA

Newborns-specific BA
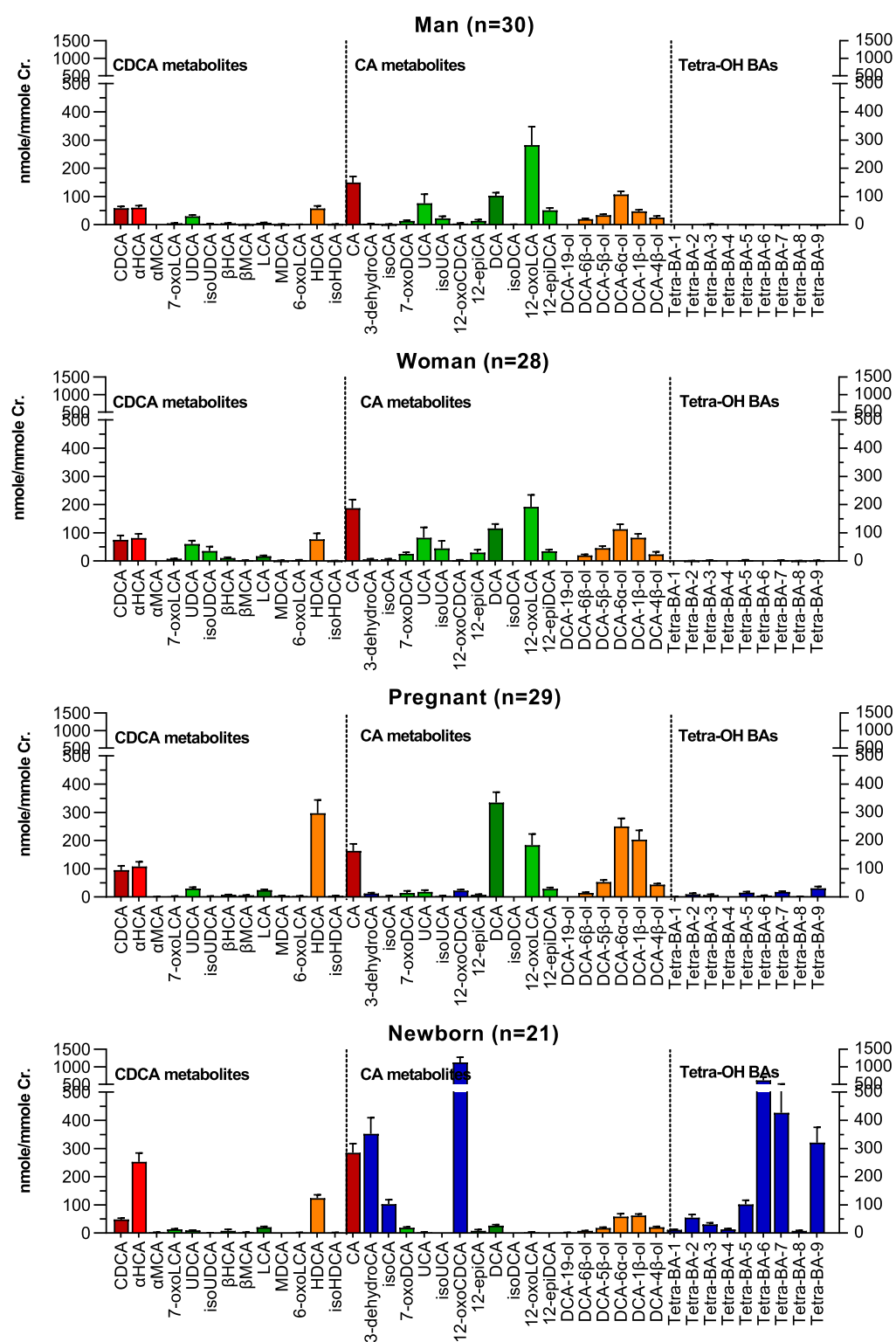

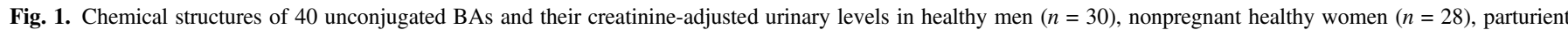
pregnant women $(n=29)$, and newborns born by cesarean section $(n=21)$. Data were shown as means \pm S.E.M.

tertiary BAs and in another way into the downstream metabolite of CDCA or CA. The urinary BA profiles of newborns were different from adult populations as expected: the primary BAs were elevated because of the decreased secondary BAs in urine. This phenomenon was more significant in the downstream metabolism of CA than in that of CDCA. Typical examples may be seen along the pathway from CA to DCA and from DCA to 12-epiDCA. However, the urinary levels of tertiary BAs, including the $6 \alpha$-hydroxylated metabolite of LCA (HDCA; $3 \alpha, 6 \alpha$-dihydroxy-5 $\beta$-cholan-24-oic acid) and the hydroxylated metabolites of DCA (DCA-1 $\beta$-ol, DCA- $6 \alpha$-ol, DCA- $5 \beta$-ol, and DCA- $4 \beta$-ol), were not elevated in newborns, as expected. Interestingly, most of them were significantly increased in the parturient pregnant women compared with healthy nonpregnant women. DCA-19-ol, the in vitro marker metabolite of fetal-specific CYP3A7, was still not appreciably detected in any populations. Moreover, newborns had significantly elevated urinary levels of the $6 \alpha$-hydroxylated metabolite of CDCA ( $\alpha \mathrm{HCA})$ and three oxidized/epimerized metabolites of CA (3-dehydroCA, isoCA, and 12-oxoCDCA). Similarly, a series of Tetra-BAs represented by five major ones (Tetra-BA-2, Tetra-BA-5 7, and Tetra-BA-9) were found as the newborn-specific BAs. These Tetra-BAs, 3-dehydroCA, and 12-oxoCDCA were also found with significantly elevated levels in parturient pregnant women compared with nonpregnant healthy women, indicating that fetal-specific BAs may have an impact on the maternal BA profiles.

Urinary BA Profiles of Newborns is Characterized by Elevated Oxidations of Primary BAs. According to the known metabolism pathways of primary, secondary, and tertiary BAs in human (Fig. 4A), the urinary metabolite ratios were calculated for the tested populations and illustrated in Fig. 4B. In this way, the difference in the downstream metabolism of CA was particularly highlighted. The decreased secondary BA metabolism of newborns was clearly shown by the significantly 
A
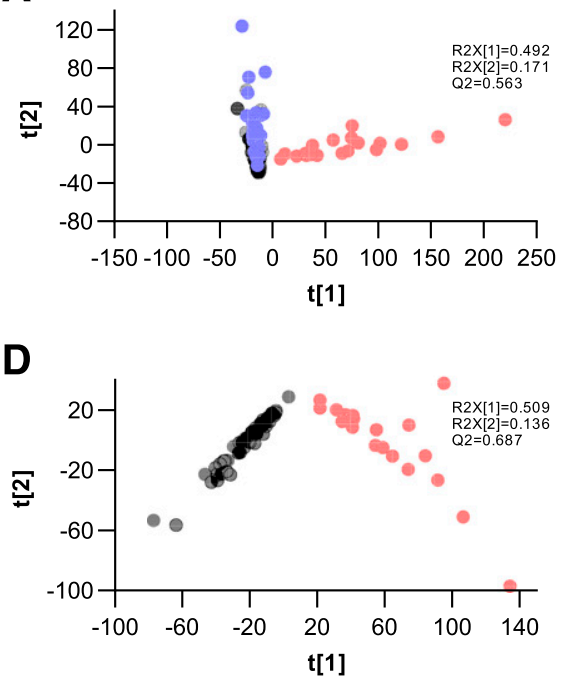

B

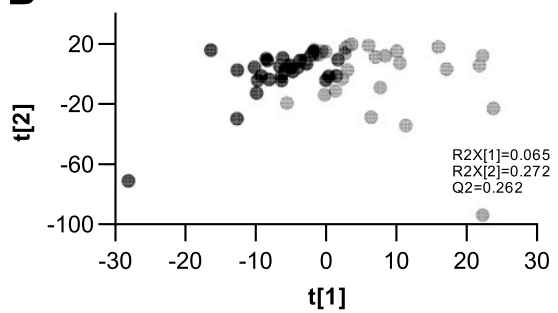

$\mathbf{E}$

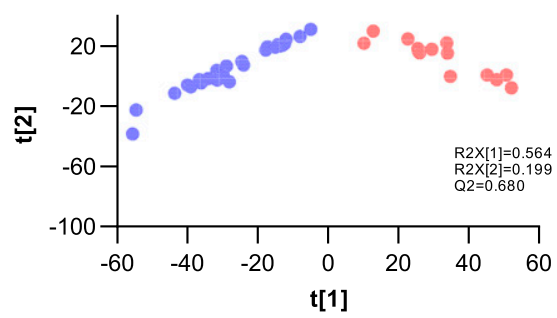

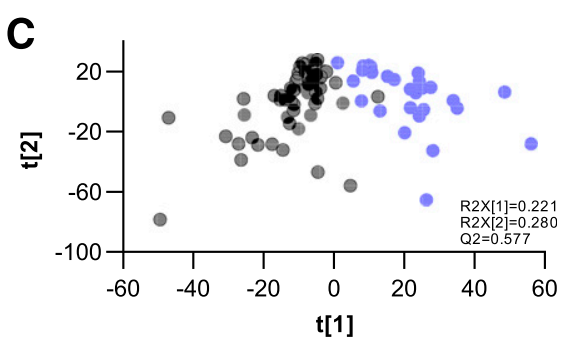

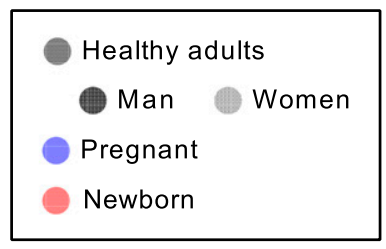

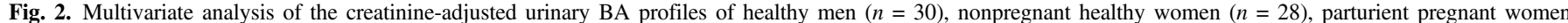

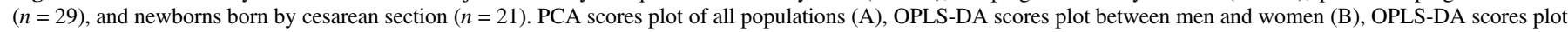

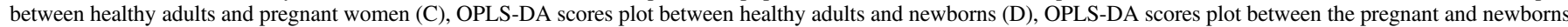

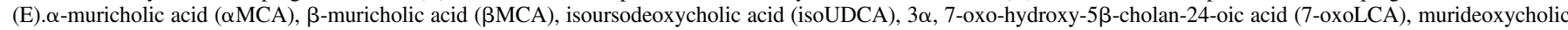

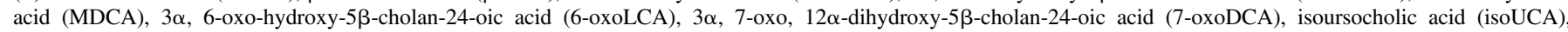
isodeoxycholic acid (isoDCA).

lower ratios of DCA/CA, UCA $(3 \alpha, 7 \beta, 12 \alpha$-trihydroxy-5 $\beta$-cholan-24-oic acid)/CA, 12-epiCA/CA, 12-oxoLCA/DCA, and 12-epiDCA/DCA compared with those of adults. Although the urinary levels of tertiary BAs were not elevated, an enhanced tertiary BA metabolism in newborns was shown by the significantly higher ratios of DCA- $6 \alpha$-ol/DCA, DCA- $1 \beta$-ol/DCA, DCA- $5 \beta$-ol/DCA, and DCA- $4 \beta$-ol/DCA compared with those of adults. For the downstream metabolism of CDCA, a decreased secondary BA metabolism was also manifested by the significantly lower ratio of UDCA/CDCA compared with that of adults. However, the ratio of LCA/CDCA of newborns was higher than that of adults, which was possibly associated with the decreased ratio of HDCA/LCA in newborns. This observation indicated that the microbial $7 \alpha$-dehydroxylation and hepatic oxidation of the $7 \alpha$-dehydroxylated metabolite may have some differences between CDCA and CA.

The metabolite ratios illustrated in Fig. 4B clearly showed that newborns have an increased oxidation metabolism of primary BAs compared with adult populations. The ratio of $\alpha \mathrm{HCA} / \mathrm{CDCA}$ of newborns was significantly higher than that of adults, indicating that newborns had a strong CYP3A-mediated $6 \alpha$-hydroxylation activity toward CDCA (Deo and Bandiera, 2008). The ratios of 3-dehydroCA/ $\mathrm{CA}$, isoCA/CA, and 12-oxoCDCA/CA, signatures of the dehydrogenation and epimerization of $\mathrm{CA}$, were also significantly higher than those of adults. Moreover, the elevated oxidation of primary BAs in newborns was most manifested by the newborn-specific Tetra-BAs. As shown in Fig. 4A, the metabolism pathways for Tetra-BAs were believed to be associated with oxidations of trihydroxy-cholan-24-oic acids, such as $\mathrm{CA}$ and $\alpha \mathrm{HCA}$. However, the ratios of them to their precursors were not calculated, because it was unknown whether they were derived from CA, $\alpha \mathrm{HCA}$, or the other trihydroxy-cholan-24-oic acids. According to the known ontogeny of CYP3A, we deduced that the fetal-specific CYP3A7 participated in the oxidation of primary BAs, particularly in the oxidation of CA and related metabolites.

Infant Liver Microsomes Have Age-Dependent Activities toward DCA 19-Oxidation. The postnatal change from CYP3A7 toward CYP3A4 occurs rapidly in the 1st month and progressively during the 1st year after birth (Saghir et al., 2012). Figure 4 showed an enhanced tertiary BA metabolism of newborns, which was manifested by the significantly higher urinary ratios of DCA- $6 \alpha$-ol/DCA, DCA- $1 \beta$-ol/DCA, DCA- $5 \beta$-ol/DCA, and DCA- $4 \beta$-ol/DCA. This observation was consistent with the fetal CYP3A7 being more active for these reactions than CYP3A4 predominating in adult livers (Chen et al., 2019; Zhang et al., 2019). We therefore compared the DCA oxidation kinetics acquired in infant liver microsomes from eight donors within 1 month and 3 years old to those obtained in liver microsomes from 14 adult donors. The liver microsomes of newborns were not included, because of limited commercial sources. The Hill coefficients fitted within the substrate (DCA) range of 1-300 $\mu \mathrm{M}$ were shown in Supplemental Table 5. According to the relative activities of CYP3A7 to CYP3A4 toward DCA oxidations, $\mathrm{C}-19>\mathrm{C}-4 \beta>\mathrm{C}-6 \alpha>\mathrm{C}-1 \beta>\mathrm{C}-5 \beta>\mathrm{C}-6 \beta$ (Chen et al., 2019), Fig. 5 illustrated the age-dependent intrinsic clearance data acquired in all the tested liver microsomes. The CYP3A7-preferring reactions to some extent showed an age-dependent decreasing tendency, such as DCA $4 \beta$-oxidation, DCA $6 \alpha$-oxidation, and DCA $1 \beta$-oxidation. The clearest age-dependent decrease of activities was observed for DCA 19-oxidation, the validated in vitro probe reaction of CYP3A7 (Chen et al., 2019). The 1-month-old infant liver microsomes with the highest CYP3A7 activity were therefore used to investigate whether the metabolic enzymes in infant liver participate in CA oxidations to produce the newborn-specific Tetra-BAs.

Infant Liver Microsomes Oxidize CA More Than Adult Liver Microsomes. In vitro oxidation metabolism of CA was investigated to clarify whether the fetal-specific Tetra-BAs are hepatic oxidized metabolites of primary BAs. Incubations of CA, TCA, and GCA were performed in parallel because the taurine conjugates of BAs predominated in newborns (Poley et al., 1964). We first compared the oxidation activities of 1-month-old infant liver microsomes (HLM-IF) and human liver microsomes from 150 (76 female and 74 male) mixed-gender pooled donors (HLM) to mimic the hepatic metabolism of newborns and adults. Figure 6A showed the metabolite formations after incubations of CA, TCA, and GCA at $50 \mu \mathrm{M}$ in HLM and HLM-IF. The oxidized metabolites of GCA and TCA were detected after being digested by cholylglycine hydrolase (Zhu et al., 2018). Four oxidative metabolites of CA were detected in the incubation 


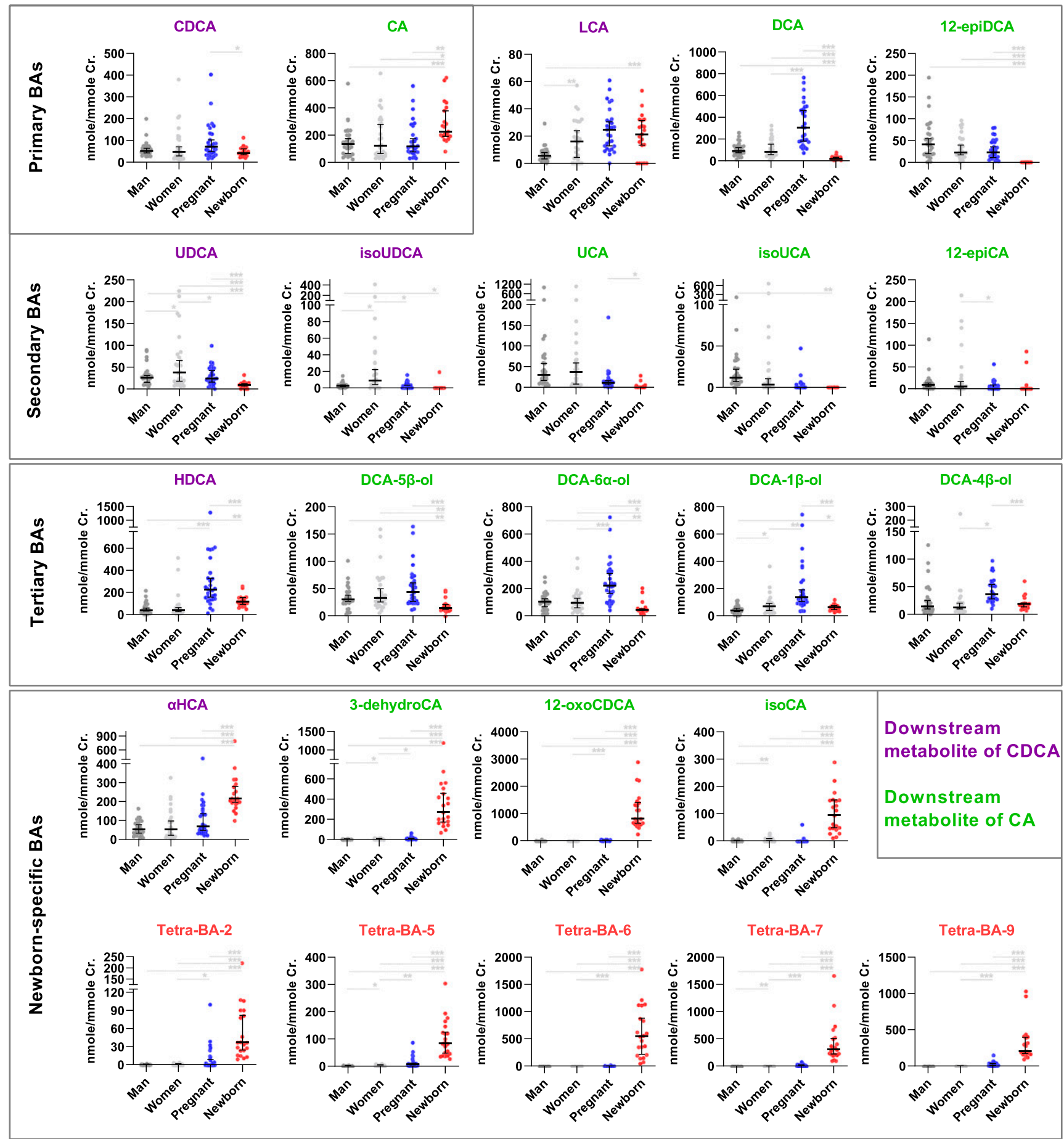

Fig. 3. The creatinine-adjusted urinary levels of representative unconjugated BAs in healthy men $(n=30)$, nonpregnant healthy women $(n=28)$, parturient pregnant women $(n=29)$, and newborns born by cesarean section $(n=21)$. Data were shown as medians with 95\% confidential interval. Unpaired $t$ tests were performed between populations $(* P<0.05 ; * * P<0.01 ; * * * P<0.001)$.isoursodeoxycholic acid (isoUDCA), isoursocholic acid (isoUCA).

samples, including Tetra-BA-2, Tetra-BA-3, Tetra-BA-5, and Tetra-BA-7, whose retention times matched well with those detected in the urines of newborns (Supplemental Table 6). Anyway, these Tetra-BAs were minor metabolites compared with the dehydrogenated metabolite, 3-dehyroCA, which was the major in vitro metabolite of CA, TCA, and GCA. The 3-dehydrogenation mechanism were considered to be involved with a $3 \alpha, 3 \beta$-diol intermediate formed by $3 \beta$-oxidation according to the 3 -dehydrogenation mechanism in DCA oxidation (Bodin et al., 2005; Zhang et al., 2019). The other fetal-specific Tetra-BAs,
12-oxoCDCA and isoCA, were not detected in the in vitro incubation samples, indicating that they may not be the hepatic metabolites of CA, TCA, and GCA.

As shown in Fig. 6A, HLM-IF exhibited much higher activities than HLM toward the oxidation reactions to produce Tetra-BA-2, Tetra-BA-3, and Tetra-BA-5. For these reactions, both HLM-IF and HLM showed higher oxidation activities toward TCA than CA and GCA. These data partly explained why the three Tetra-BAs were detected in newborns but were not found in adults because CA is mainly conjugated with 
A

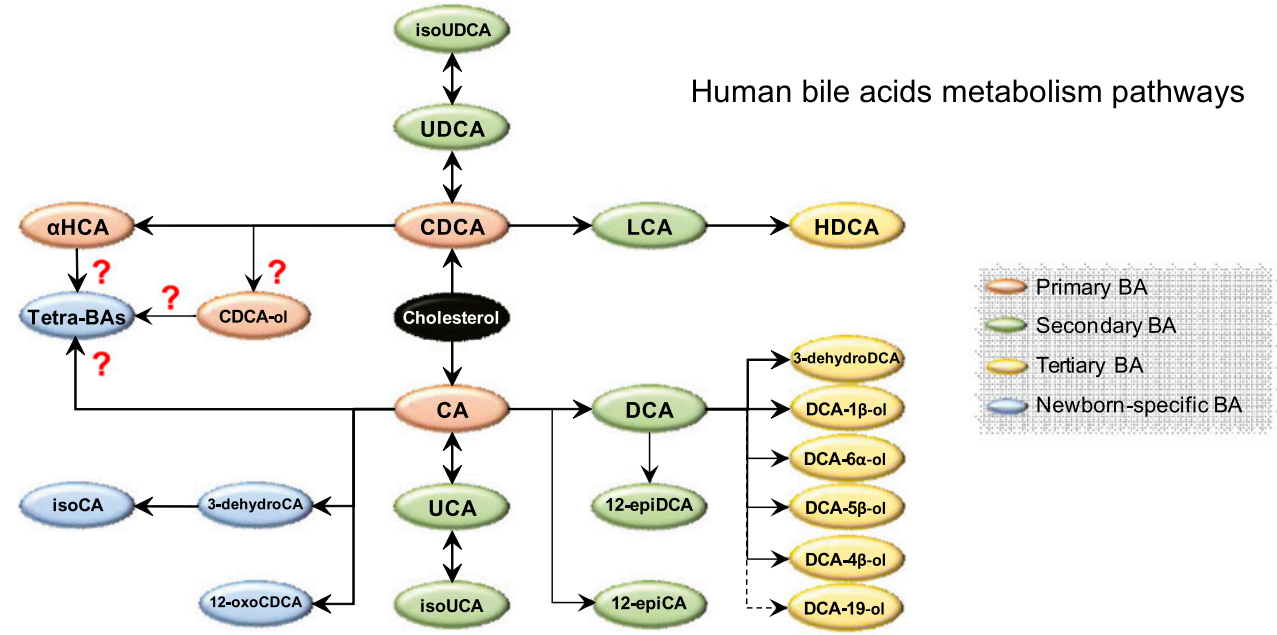

B

LCA/CDCA

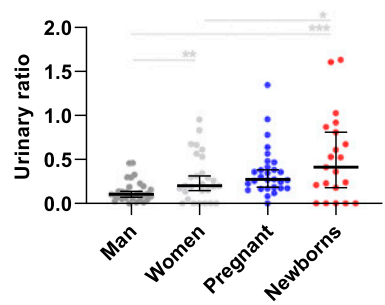

DCA/CA

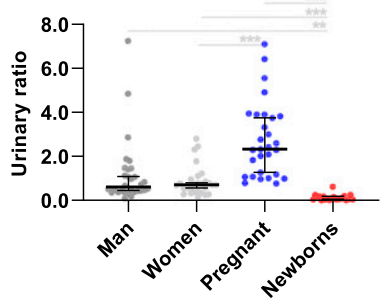

12-epiCA/CA

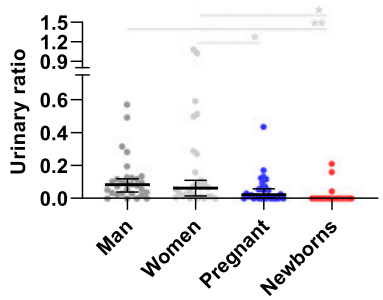

12-epiDCA/DCA

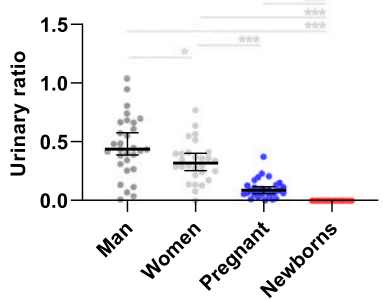

UDCA/CDCA

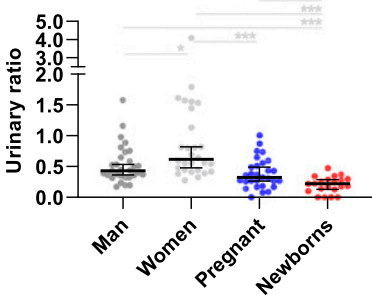

UCA/CA

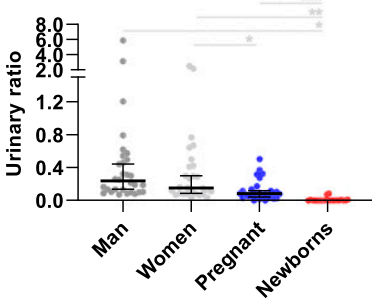

12-oxoLCA/DCA

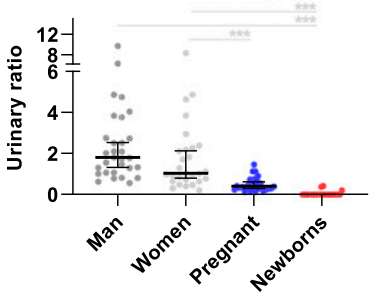

3-dehydroCA/CA

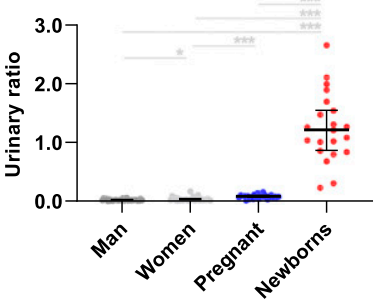

HDCA/LCA

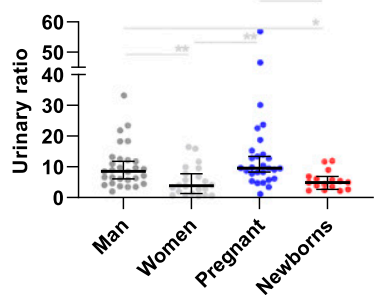

DCA-6a-ol/DCA

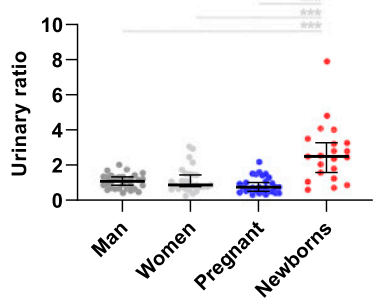

DCA-5 $\beta$-ol/DCA

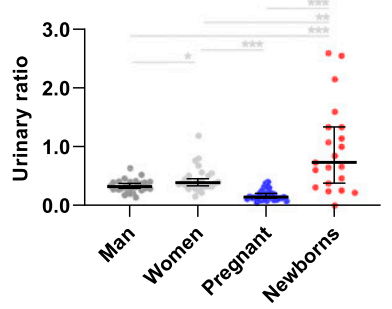

isoCA/CA

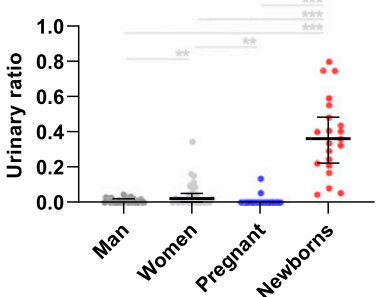

aHCA/CDCA

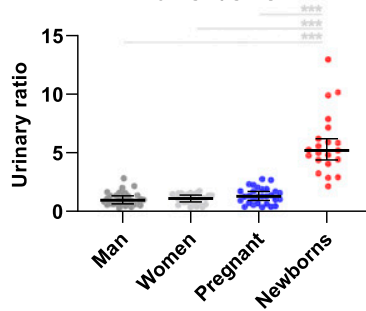

DCA-1 $\beta$-ol/DCA

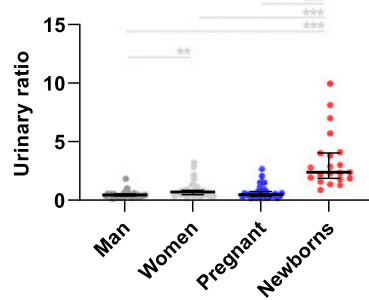

DCA-4ß-ol/DCA

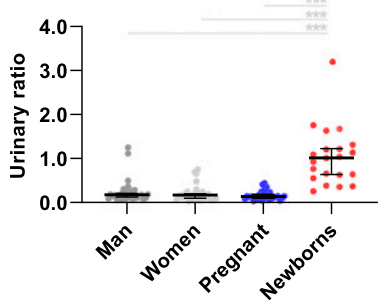

12-oxoCDCA/CA

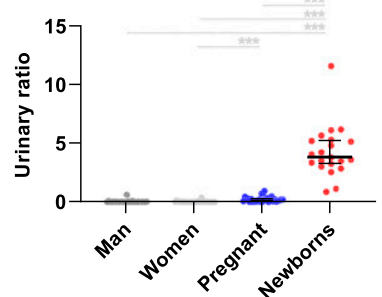

Fig. 4. Metabolic pathways of bile acids in human (A) and their urinary ratios in healthy men $(n=30)$, nonpregnant healthy women $(n=28)$, parturient pregnant women $(n=29)$, and newborns born by cesarean section $(n=21)$. Data were shown as medians with $95 \%$ confidential interval. Unpaired $t$ tests were performed between populations $(* P<0.05 ; * * P<0.01 ; * * P<0.001)$.isoursodeoxycholic acid (isoUDCA), isoursocholic acid (isoUCA). 

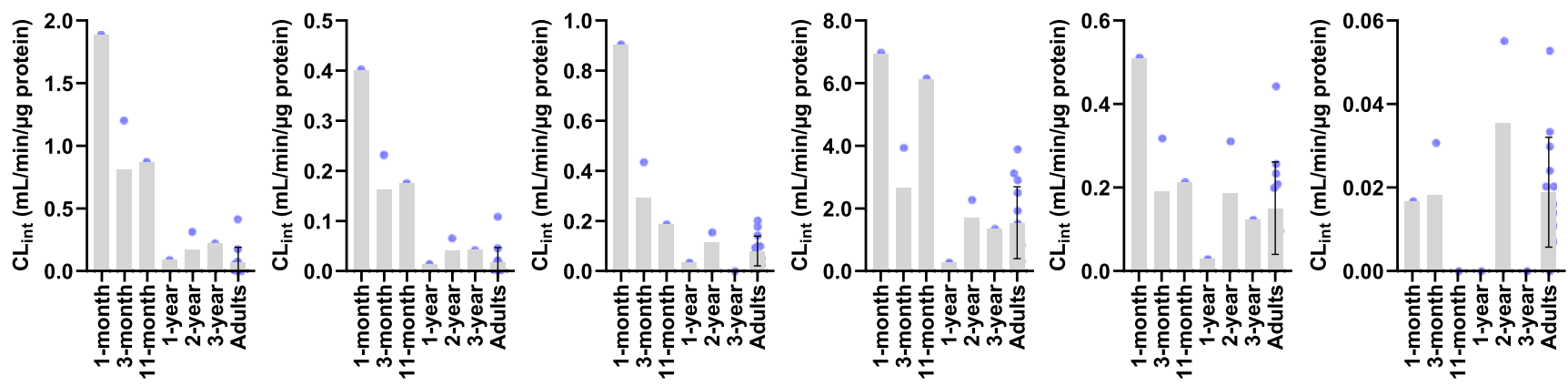

Donor age

Fig. 5. The in vitro intrinsic clearance $\left(\mathrm{CL}_{\text {int }}\right)$ of DCA oxidations in the infant liver microsomes from eight single donors from 1 month to 3 years old and the adult liver microsomes from 14 single donors. Data were shown as means \pm S.D. for adult liver microsomes.

taurine in newborns. However, HLM-IF and HLM showed comparable activities toward the $3 \beta$-oxidation of TCA and GCA, as well as the oxidation reactions to produce Tetra-BA-7. Therefore, the in vitro metabolism assay in liver microsomes did not explain the fetalspecific urinary excretion of 3-dehydroCA and Tetra-BA-7 compared with adults. Further studies should consider whether the intestinal epithelia or infant gut microbes contribute to the fetal-specific oxidative metabolism of primary BAs.

Limited Contributions of Recombinant CYP3A7 to Cholate Oxidations. To clarify whether CYP3A7 in HLM-IF contributes to the oxidation reactions to produce Tetra-BA-2, Tetra-BA-3, and Tetra-BA-5, we subsequently compared the oxidation activities of recombinant CYP3A4, 3A5, and 3A7 enzymes for CA, TCA, and GCA (Fig. 6B). The recombinant $\mathrm{P} 450$ enzyme assay did not show consistency with the observations in HLM-IF and HLM, except for the $3 \beta$-oxidation, toward which CYP3A4, 3A5, and 3A7 had comparable activities for the substrates of TCA and GCA to produce 3-dehydrogenated metabolites. Comparable activities of CYP3A isoenzymes had also been shown toward the $3 \beta$-oxidation of glycodeoxycholate and taurodeoxycholate to produce 3-dehydrogenated metabolites (Zhang et al., 2019). Compared with CYP3A4 and 3A7, CYP3A5 had no or trace activity for the oxidative reactions to produce Tetra-BAs. However, CYP3A7 did not exhibit stronger oxidation activities than CYP3A4 as expectated for the oxidation reactions to produce Tetra-BA-2, Tetra-BA-3, and Tetra-BA-5, indicating that the fetal-specific CYP3A7 may play a limited role in the oxidation metabolism of CA, TCA, and GCA in newborns.

\section{Discussion}

It has been known for a long time that the BA metabolism of infants is different from that of adults (Poley et al., 1964). Literature published decades before had found a series of fetal-specific BAs with "unusual" oxidation sites, including $\mathrm{C}-1 \beta,-2 \beta,-4 \beta,-6 \alpha / \beta$, and -19 , in early human life. A summary of this literature may be seen in a recent book (Sjövall et al., 2010). However, it remains undefined how and to what extent the fetal BA metabolism is different from that of adults along the axis of primary, secondary, and tertiary BAs. Accordingly, the following questions are still unknown: What is the driving force for the transition from the neonatal BA metabolism phenotype to that of adults? What is the driving force for the ontogeny of P450s that play important roles in both lipid metabolism and pediatric pharmacology? And what is the relationship between the development of human BA's host-gut microbial co-metabolism and metabolic diseases during pregnancy and childhood? In this regard, revisiting studies of the perinatal BA metabolism is imperative in the era of metagenomics. Such results obtained in murine animals should be carefully extrapolated to humans because they have BA metabolism pathways markedly different from those in humans (Thakare et al., 2018a,b; Lin et al., 2020). In human studies, however, samples of newborns and infants are available mainly in diaper disposals because of ethical and practical issues. Meconium is hard to analyze precisely because it rapidly loses water on diapers and contaminates the urine samples. We therefore developed and validated a protocol to isolate the diaper urine samples without meconium contamination. The $\mathrm{CaCl}_{2}$ method showed high efficiency and acceptable accuracy to extract urinary BAs absorbed in the diaper gel. Using this method, we were able to compare the urinary BA profiles of newborns to those of adult controls. Newborns born by cesarean section were enrolled in this work because it is hard to collect diapers from babies born naturally, who usually leave the hospital 24 hours after delivery.

By using a BA profiling technique in assistance with the in vitro metabolism assays, this work disclosed that the urinary BA profiles of newborns are characterized by the elevated oxidative metabolism of primary BAs compared with those of adults. A deeper understanding of the difference in BA conjugation metabolism relies on development of the next-generation BA profiling techniques, including not only $\mathrm{N}$-amidated forms but also sulfated and glucuronidated forms of BA. This metabolic phenotype seemed to be more significant in the downstream of CA, which was manifested by the significantly elevated urinary ratios of 3-dehydroCA/CA, isoCA/CA, and 12-oxoCDCA/CA than that of CDCA, which was indicated by the significantly increased urinary ratios of $\alpha \mathrm{HCA} / \mathrm{CDCA}$. High levels of 3-dehydroCA have been previously reported in amniotic fluid of pregnant women (Nakagawa and Setchell, 1990) and urine of newborns (Kimura et al., 1997) and preterm and full-term infants (Maeda et al., 2003). Moreover, such a phenotype was more clearly shown by the nine fetal-specific Tetra-BAs, which have been mentioned decades before in urine of patients with hepatobiliary diseases (Alme et al., 1977) and newborns (Strandvik and Wikström, 1982). Among these Tetra-BAs, four metabolites (Tetra-BA-2, Tetra-BA-3, Tetra-BA-5, and Tetra-BA-7) were oxidized metabolites of CA in infant liver microsomes. Metabolism pathways for the other Tetra-BAs were believed to be associated with hepatic or extrahepatic oxidations of the major trihydroxy-cholan-24-oic acids of newborns. Chemical structures of these Tetra-BAs were not identified in this work, because of time-consuming synthesis of the potential authentic standards. According to related literature (Tohma et al., 1985; Kimura et al., 1994), the major Tetra-BAs were believed to be associated with 


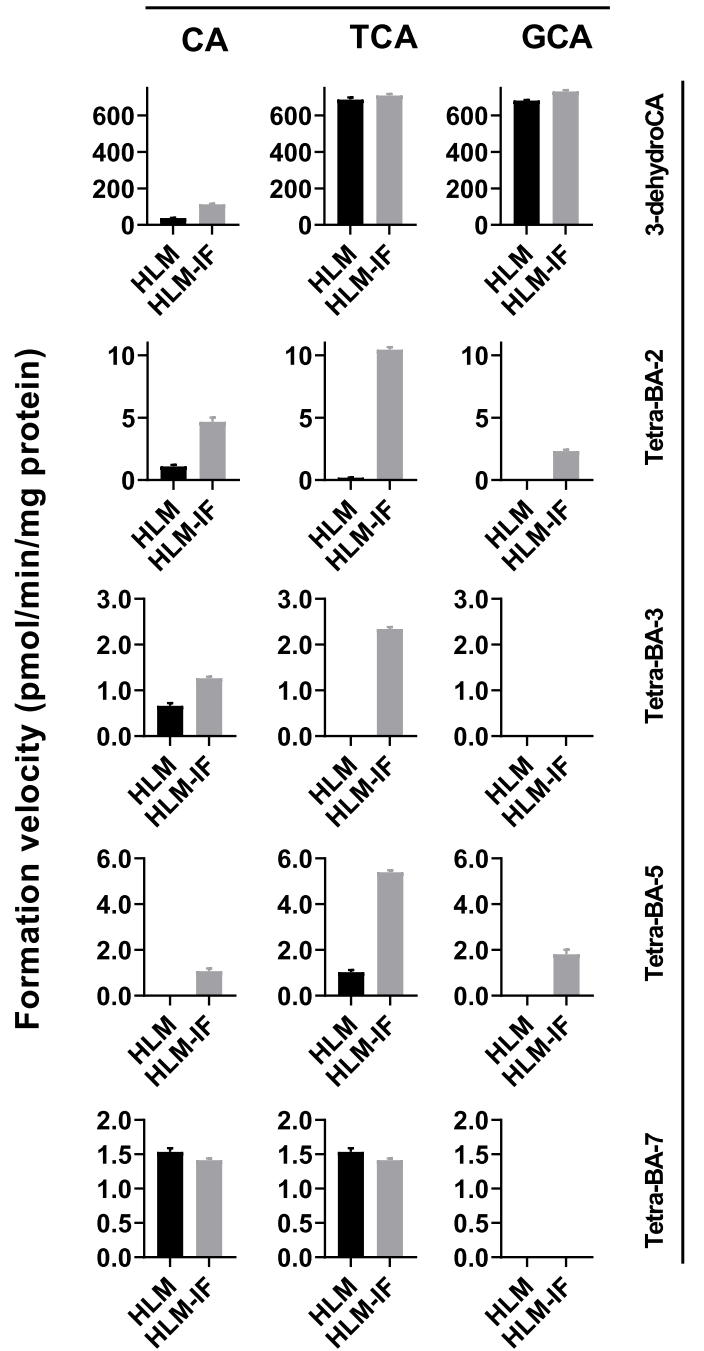

B
Substrates

CA TCA GCA

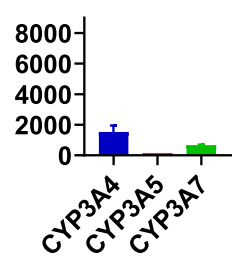

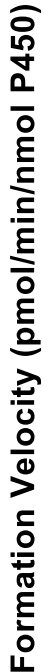
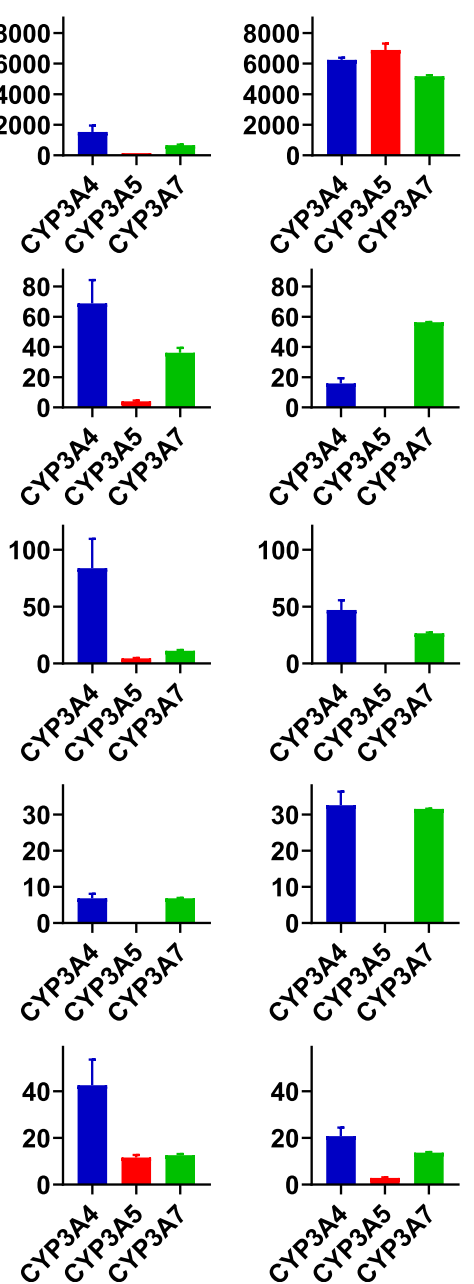

Fig. 6. In vitro metabolite formation after incubation of CA, TCA, and GCA at $50 \mu \mathrm{M}$ in liver microsomes (A) and recombinant CYP3A enzymes (B) for 60 minutes. Data were shown as means \pm S.D. $(n=3)$ in picomoles per minute per milligram protein for adult liver microsomes (HLM) and infant liver microsomes (HLM-IF), which were incubated at a protein level of $0.5 \mathrm{mg} / \mathrm{ml}$, and in picomoles per minute per nanomoles P450 for the recombinant CYP3A4, 3A5, and 3A7 enzymes incubated at a protein level of $50 \mathrm{pmol} \mathrm{protein} / \mathrm{ml}$.

the $1 \beta$-hydroxylated metabolites of CA or the subsequent hydroxylated metabolites of the oxidized metabolites of CDCA, such as $\alpha \mathrm{HCA}$ and CDCA- $1 \beta$-ol. The other Tetra-BAs were proposed to be metabolites with oxidation sites on the A or B ring (Dumaswala et al., 1989; Kurosawa et al., 1989), which is similar to those of DCA disclosed in our publications (Chen et al., 2019; Zhang et al., 2019).

The in-depth analysis of urinary BA profiles also found a decreased secondary BA metabolism in newborns, which coincided with the elevated oxidation of primary BAs because newborns have limited colonization of gut bacteria responsible for secondary BA production. Accordingly, the tertiary BA metabolism, which may play a complementary role in the disposition of secondary BA in adults, also did not contribute significantly to the renal excretion of BAs in newborns, even though the newborns exhibited enhanced tertiary BA metabolism, which was indicated by the elevated ratios of DCA- $6 \alpha$-ol/DCA, DCA- $1 \beta$-ol/DCA, DCA- $5 \beta$-ol/DCA, and DCA- $4 \beta$-ol/DCA. The phenotype of increased tertiary BA metabolism is consistent with newborns having much higher hepatic activities of fetal CYP3A7, which has stronger activity toward DCA oxidation than CYP3A4, which dominates in adult livers. DCA-19-ol, the major oxidized metabolite of DCA in infant microsomes (Fig. 5), was also not appreciably detected in urine samples of newborns as expected. The in vitro/in vivo inconsistency of DCA 19-hydroxylation reinforced our previous deduction about the subsequent conversion of DCA-19-ol into another unknown metabolite (Chen et al., 2019; Zhang et al., 2019). It has to be mentioned once again that newborns born by cesarean section rather than natural birth were enrolled in this work. It is known that the delivery method plays a decisive role in the first colonization of gut bacteria in newborns and the subsequent developments of gut microbiota in infants (Chu et al., 2017). Such a disparity in delivery mode may result in different secondary BA metabolism and the subsequent tertiary BA metabolism. Future works are needed to decipher the phenotypic difference of BA metabolism between newborns born by cesarean section and newborns born naturally.

According to the BA metabolism phenotype of newborns born by cesarean section, we believed that the hepatic oxidations of primary BAs might have played a vital role in BA elimination in early life. This deduction was also evident by the fact that these fetal-specific BAs were 
significantly elevated in urine of parturient pregnant women compared with nonpregnant healthy women. The examples included all the TetraBAs, DCA- $6 \alpha$-ol (tertiary BA), DCA- $1 \beta$-ol (tertiary BA), DCA- $4 \beta$-ol (tertiary BA), 3-dehydroCA (fetal-specific BA), and 12-oxoCDCA (fetal-specific BA) in the downstream metabolism of CA, as well as HDCA (tertiary BA) in the downstream metabolism of CDCA. The significantly elevated urinary excretion of these BAs in parturient pregnant women indicated that oxidized metabolites of primary BAs and the tertiary BA metabolites produced by fetus may be excreted into amniotic fluid through meconium and fetal urine for maternal disposition. Simultaneous analysis of the BA profiles in maternal amniotic fluid, urine, and feces in comparison with meconium and urine of newborns and infants may provide fundamental information about whether such a maternal-fetal interplay of BA disposition is associated with perinatal cholestasis-associated diseases.

This work performed preliminary in vitro metabolism studies to investigate whether the fetal-specific CYP3A7 contributes to the elevated oxidative metabolism of primary BA in newborns, exemplified by the substrates of CA, TCA, and GCA. The HLM-IF from a 1-monthold donor, which was characterized by DCA 19-oxidation to have the highest CYP3A7 activities (Chen et al., 2019), was employed as an alternative of newborn liver fractions. HLM-IF exhibited much higher activities than HLM of pooled adult donors, particularly toward the oxidation reactions of TCA to produce Tetra-BA-2, Tetra-BA-3, and Tetra-BA-5. It is unclear to what extent the difference occurring in human intestine microsomes is due to the lack of commercial sources of infant intestine microsomes. However, beyond our expectations, the recombinant enzyme assays did not show that CYP3A7 significantly contributed to these reactions compared with CYP3A4. CYP3A5 showed no or trace activities to these reactions, except for the 3-dehydrogenation reaction of TCA and GCA. We therefore concluded that the fetal-specific CYP3A7 might have played a limited role in the oxidation metabolism of CA, TCA, and GCA in newborns. Since most drug-metabolizing P450 isoforms, including CYP1A2, CYP2A6, CYP2B6, CYP2D6, CYP2C9, CYP2C19, CYP1E2, and CYP3A4, express limitedly in newborn livers and have a similar postnatal ontogenic pattern (Hines, 2007; Upreti and Wahlstrom, 2016; Allegaert and van den Anker, 2019), it is deduced that some other fetal-specific $\mathrm{P} 450$ isoforms may participate in the oxidations of CA, GCA, and particularly TCA.

In conclusion, this work demonstrated how the BA metabolism of newborns born by cesarean section differs from that of adults along the axis of primary, secondary, and tertiary BAs. Because of a lack of developed gut microbiota, newborns have poor secondary BA metabolism, which plays a vital role in the BA disposition of adults. Subsequently, the tertiary metabolism of secondary BAs contributes limitedly to the disposition of BAs in newborns. As a result, the primary BAs synthesized in the infant liver undergo extensive oxidation metabolism, resulting in significantly elevated levels of the oxidized metabolites in newborn urine, such as $\alpha \mathrm{HCA}, 3$-dehydroCA, isoCA, and 12-oxoCDCA, and several Tetra-BAs, some of which were characterized as hydroxylated metabolites of CA by in vitro metabolism assay in infant liver microsomes. Compared with CYP3A4, the fetal-specific CYP3A7 did not contribute to CA oxidation as much as expected. It is therefore deduced that the fetal-specific BA disposition pattern is gradually translated into the metabolism axis of primary, secondary, and tertiary BAs of adults with the developments of gut microbiota and the ontogeny of P450 enzymes after birth. Comprehensive technologies involving metagenomics, transcriptomics, proteomics, metabolomics, and in vitro activity assays may be helpful to decipher the mechanism underlying the fetal-specific BA metabolism phenotype and its transition to the adult phenotype. Such knowledge is of particular importance in both pediatric pharmacology and molecular pathology of perinatal diseases, such as perinatal cholestasis diseases, and promisingly, is linked to other ontogeny and development, since BAs play important roles in lipid absorption and regulation of lipid/carbohydrate metabolism.

\section{Acknowledgments}

We are grateful to Dr. Liang Xu (West China School of Pharmacy, Sichuan University) for his support of bile acid synthesis.

\section{Authorship Contributions}

Participated in research design: Lan.

Conducted experiments: W.-X. Wang, L. Chen, G.-Y. Wang, J.-L. Zhang, Tan, Lin, Y.-J. Chen, J. Zhang, Zhu, Miao, Su.

Performed data analysis: W.-X. Wang, L. Chen, G.-Y. Wang, Lan.

Wrote or contributed to the writing of the manuscript: W.-X. Wang, L. Chen, Liu, Jia, Lan.

\section{References}

Allegaert K and van den Anker J (2019) Ontogeny of phase I metabolism of drugs. J Clin Pharmacol 59 (Suppl 1):S33-S41.

Almé B, Bremmelgaard A, Sjövall J, and Thomassen P (1977) Analysis of metabolic profiles of bile acids in urine using a lipophilic anion exchanger and computerized gas-liquid chromatorgaphy-mass spectrometry. J Lipid Res 18:339-362.

Almé B and Sjövall J (1980) Analysis of bile acid glucuronides in urine. Identification of 3 alpha, 6 alpha, 12 alpha-trihydroxy-5 beta-cholanoic acid. J Steroid Biochem 13:907-916.

Batta AK, Arora R, Salen G, Tint GS, Eskreis D, and Katz S (1989) Characterization of serum and urinary bile acids in patients with primary biliary cirrhosis by gas-liquid chromatography-mass spectrometry: effect of ursodeoxycholic acid treatment. J Lipid Res 30:1953-1962.

Bodin K, Lindbom U, and Diczfalusy U (2005) Novel pathways of bile acid metabolism involving CYP3A4. Biochim Biophys Acta 1687:84-93.

Chen YJ, Zhang J, Zhu PP, Tan XW, Lin QH, Wang WX, Yin SS, Gao LZ, Su MM, Liu CX, et al (2019) Stereoselective oxidation kinetics of deoxycholate in recombinant and microsomal CYP3A enzymes: deoxycholate 19-hydroxylation is an in vitro marker of CYP3A7 activity. Drug Metab Dispos 47:574-581.

Chu DM, Ma J, Prince AL, Antony KM, Seferovic MD, and Aagaard KM (2017) Maturation of the infant microbiome community structure and function across multiple body sites and in relation to mode of delivery. Nat Med 23:314-326.

Dawson PA and Karpen SJ (2015) Intestinal transport and metabolism of bile acids. J Lipid Res 56:1085-1099.

Deo AK and Bandiera SM (2008) Identification of human hepatic cytochrome p450 enzymes involved in the biotransformation of cholic and chenodeoxycholic acid. Drug Metab Dispos 36:1983-1991.

Dumaswala R, Setchell KD, Zimmer-Nechemias L, Iida T, Goto J, and Nambara T (1989) Identification of 3 alpha, 4 beta,7 alpha-trihydroxy- 5 beta-cholanoic acid in human bile: reflection of a new pathway in bile acid metabolism in humans. $J$ Lipid Res 30:847-856.

Halilbasic E, Claudel T, and Trauner M (2013) Bile acid transporters and regulatory nuclear receptors in the liver and beyond. J Hepatol 58:155-168.

Hines RN (2007) Ontogeny of human hepatic cytochromes P450. J Biochem Mol Toxicol 21: $169-175$.

Hu Y, Beach J, Raymer J, and Gardner M (2004) Disposable diaper to collect urine samples from young children for pyrethroid pesticide studies. J Expo Anal Environ Epidemiol 14:378-384.

Ikegawa S, Murai T, Matsui A, and Tohma M (1994) Radioimmunoassay of conjugated 1 betahydroxycholic acid in dried blood spots for diagnosis of congenital biliary atresia. Biol Pharm Bull 17:5-8.

Kakiyama G, Muto A, Takei H, Nittono H, Murai T, Kurosawa T, Hofmann AF, Pandak WM, and Bajaj JS (2014) A simple and accurate HPLC method for fecal bile acid profile in healthy and cirrhotic subjects: validation by GC-MS and LC-MS. J Lipid Res 55:978-990.

Kimura A, Suzuki M, Murai T, Inoue T, Kato H, Hori D, Nomura Y, Yoshimura T, Kurosawa T, and Tohma M (1997) Perinatal bile acid metabolism: analysis of urinary bile acids in pregnant women and newborns. J Lipid Res 38:1954-1962.

Kimura A, Yamakawa R, Ushijima K, Fujisawa T, Kuriya N, Kato H, Inokuchi T, Mahara R, Kurosawa T, and Tohma M (1994) Fetal bile acid metabolism during infancy: analysis of 1 betahydroxylated bile acids in urine, meconium and feces. Hepatology 20:819-824.

Kivistö KT, Bookjans G, Fromm MF, Griese EU, Münzel P, and Kroemer HK (1996) Expression of CYP3A4, CYP3A5 and CYP3A7 in human duodenal tissue. Br J Clin Pharmacol 42:387-389. Kumagai M, Kimura A, Takei H, Kurosawa T, Aoki K, Inokuchi T, and Matsuishi T (2007) Perinatal bile acid metabolism: bile acid analysis of meconium of preterm and full-term infants. I Gastroenterol 42:904-910.

Kurosawa T, Mahara R, Nittono H, and Tohma M (1989) Synthesis of 6-hydroxylated bile acids and identification of 3 alpha, 6 alpha, 7 alpha, 12 alpha-tetrahydroxy-5 beta-cholan-24-oic acid in human meconium and neonatal urine. Chem Pharm Bull (Tokyo) 37:557-559.

Kurosawa T, Nomura Y, Mahara R, Yoshimura T, Kimura A, Ikegawa S, and Tohma M (1995) Synthesis of 19-hydroxylated bile acids and identification of 3 alpha, 7 alpha, 12 alpha,19-tetrahydroxy-5 beta-cholan-24-oic acid in human neonatal urine. Chem Pharm Bull (Tokyo) 43:1551-1557.

Lin Q, Tan X, Wang W, Zeng W, Gui L, Su M, Liu C, Jia W, Xu L, and Lan K (2020) Species differences of bile acid redox metabolism: tertiary oxidation of deoxycholate is conserved in preclinical animals. Drug Metab Dispos 48:499-507.

Liu L, Xia T, Zhang X, Barr DB, Alamdar A, Zhang J, Tian M, Huang Q, and Shen H (2014) Biomonitoring of infant exposure to phenolic endocrine disruptors using urine expressed from disposable gel diapers. Anal Bioanal Chem 406:5049-5054.

Maeda K, Kimura A, Yamato Y, and Matsuishi T (2003) Perinatal bile acid metabolism: analysis of urinary unsaturated ketonic bile acids in preterm and full-term infants. Acta Paediatr 92:216-220 
Mahara R, Takeshita H, Kurosawa T, Ikegawa S, and Tohma M (1987) Determination of 1.BETA.hydroxylated bile acids and related compounds in human biological fluids by gas chromatography-mass spectrometry. Anal Sci 3:449-452.

Meng LJ, Reyes H, Palma J, Hernandez I, Ribalta J, and Sjövall J (1997a) Effects of ursodeoxycholic acid on conjugated bile acids and progesterone metabolites in serum and urine of patients with intrahepatic cholestasis of pregnancy. J Hepatol 27:1029-1040.

Meng LJ, Reyes H, Palma J, Hernandez I, Ribalta J, and Sjövall J (1997b) Profiles of bile acids and progesterone metabolites in the urine and serum of women with intrahepatic cholestasis of pregnancy. J Hepatol 27:346-357.

Miyara T, Shindo N, Tohma M, and Murayama K (1990) Capillary gas chromatography/ negative ion chemical ionization mass spectrometry for the quantification of bile acids including 1 beta-hydroxylated and unsaturated bile acids in serum and urine. Biomed Chromatogr 4:56-60.

Nakagawa M and Setchell KD (1990) Bile acid metabolism in early life: studies of amniotic fluid. J Lipid Res 31:1089-1098.

Nishiura H, Kimura A, Yamato Y, Aoki K, Inokuchi T, Kurosawa T, and Matsuishi T (2010) Developmental pattern of urinary bile acid profile in preterm infants. Pediatr Int 52:44-50.

Poley JR, Dower JC, Owen CA Jr., and Stickler GB (1964) Bile acids in infants and children. J Lab Clin Med 63:838-846.

Russell DW (2003) The enzymes, regulation, and genetics of bile acid synthesis. Annu Rev Biochem 72:137-174.

Saghir SA, Khan SA, and McCoy AT (2012) Ontogeny of mammalian metabolizing enzymes in humans and animals used in toxicological studies. Crit Rev Toxicol 42:323-357.

Schuetz JD, Kauma S, and Guzelian PS (1993) Identification of the fetal liver cytochrome CYP3A7 in human endometrium and placenta. J Clin Invest 92:1018-1024.

Setchell KD, Dumaswala R, Colombo C, and Ronchi M (1988) Hepatic bile acid metabolism during early development revealed from the analysis of human fetal gallbladder bile. J Biol Chem 263: $16637-16644$.

Shoda J, Mahara R, Osuga T, Tohma M, Ohnishi S, Miyazaki H, Tanaka N, and Matsuzaki Y (1988) Similarity of unusual bile acids in human umbilical cord blood and amniotic fluid from newborns and in sera and urine from adult patients with cholestatic liver diseases. J Lipid Res $\mathbf{2 9}$ : $847-858$.

Shoda J, Osuga T, Mahara R, Tohma M, Matsuura K, Tanaka N, Matsuzaki Y, and Miyazaki H (1989) Altered metabolism of bile acids in cholestasis: determination of 1 beta- and 6 alphahydroxylated metabolites. J Chromatogr A 488:315-328.

Shoda J, Tanaka N, Osuga T, Matsuura K, and Miyazaki H (1990) Altered bile acid metabolism in liver disease: concurrent occurrence of C-1 and C-6 hydroxylated bile acid metabolites and their preferential excretion into urine. J Lipid Res 31:249-259.
Sjövall J, Griffiths WJ, Setchell KDR, Mano N, and Goto J (2010) Analysis of Bile Acids, Springer, New York.

Strandvik B and Wikström SA (1982) Tetrahydroxylated bile acids in healthy human newborns. Eur J Clin Invest 12:301-305.

Suzuki M, Murai T, Yoshimura T, Kimura A, Kurosawa T, and Tohma M (1997) Determination of 3-oxo-delta4- and 3-oxo-delta4,6-bile acids and related compounds in biological fluids of infants with cholestasis by gas chromatography-mass spectrometry. J Chromatogr B Biomed Sci Appl 693: $11-21$.

Thakare R, Alamoudi JA, Gautam N, Rodrigues AD, and Alnouti Y (2018a) Species differences in bile acids I. Plasma and urine bile acid composition. J Appl Toxicol 38:1323-1335.

Thakare R, Alamoudi JA, Gautam N, Rodrigues AD, and Alnouti Y (2018b) Species differences in bile acids II. Bile acid metabolism. J Appl Toxicol 38:1336-1352.

Tohma M, Mahara R, Takeshita H, Kurosawa T, and Ikegawa S (1986) Synthesis of the 1 beta-hydroxylated bile acids, unusual bile acids in human biological fluids. Chem Pharm Bull (Tokyo) 34:2890-2899.

Tohma M, Mahara R, Takeshita H, Kurosawa T, Ikegawa S, and Nittono H (1985) Synthesis of the 1 beta-hydroxylated bile acids and identification of 1 beta, 3 alpha, 7 alpha-trihydroxy- and 1 beta, 3 alpha,7 alpha, 12 alpha-tetrahydroxy-5 beta-cholan-24-oic acids in human meconium. Chem Pharm Bull (Tokyo) 33:3071-3073.

Upreti VV and Wahlstrom JL (2016) Meta-analysis of hepatic cytochrome P450 ontogeny to underwrite the prediction of pediatric pharmacokinetics using physiologically based pharmacokinetic modeling. J Clin Pharmacol 56:266-283.

Yin S, Su M, Xie G, Li X, Wei R, Liu C, Lan K, and Jia W (2017) Factors affecting separation and detection of bile acids by liquid chromatography coupled with mass spectrometry in negative mode. Anal Bioanal Chem 409:5533-5545.

Zhang J, Gao LZ, Chen YJ, Zhu PP, Yin SS, Su MM, Ni Y, Miao J, Wu WL, Chen H, et al. (2019) Continuum of host-gut microbial co-metabolism: host CYP3A4/3A7 are responsible for tertiary oxidations of deoxycholate species. Drug Metab Dispos 47:283-294.

Zhu P, Zhang J, Chen Y, Yin S, Su M, Xie G, Brouwer KLR, Liu C, Lan K, and Jia W (2018) Analysis of human $\mathrm{C} 24$ bile acids metabolome in serum and urine based on enzyme digestion of conjugated bile acids and LC-MS determination of unconjugated bile acids. Anal Bioanal Chem 410:5287-5300.

Address correspondence to: Dr. Ke Lan, West China School of Pharmacy, Sichuan University, No.17 People's South Road, Chengdu 610041, China. E-mail: lanwoco@scu.edu.cn 